\title{
Finding Flow in Granular Media
}

\author{
A new model can predict how packed particles move through both 2D and \\ 3D channels.
}

By Rachel Berkowitz

$\Lambda$ hiker's misstep can trigger an avalanche on a pebbled hillside as the pebbles transition from load-bearing solid to flowing fluid. But how to predict the flows of granular systems in their steady state remains open to debate. Now, using a series of computer simulations, Seongmin Kim and Ken Kamrin of the Massachusetts Institute of Technology demonstrate a model that makes this prediction for dense granular systems [1].

In flowing granular systems, a key issue is predicting the macroscopic stress that emerges from interactions between grains. Several models predict this behavior using other physical quantities. However, the results from those models don't fit well when grains are densely packed or when they flow under gravity. For that, Kim and Kamrin propose a simple power law, which comes from thermodynamic descriptions of similar systems. Their model, which works for 2D and 3D systems, expresses the macroscopic stress in terms of the individual grains' velocity fluctuations and in terms of the average velocity changes throughout the system.

The duo conducted simulations of grains packed in 2D and 3D geometries and subjected them to various loads. Calculations of the grains' positions, velocities, and interacting forces led the researchers to conclude that the granular temperature, which describes the velocity fluctuations, softens granular materials and assists flow. Those calculations revealed that the stress that drives motion scales with the $1 / 6$ (3D) or 1/8 (2D) power of the system's granular temperature. The researchers found that the relationship holds for different channel geometries, flow speeds, packing densities, and grain surface friction.

Rachel Berkowitz is a Corresponding Editor for Physics based in Seattle, Washington, and Vancouver, Canada.

\section{REFERENCES}

1. S. Kim and K. Kamrin, "Power-law scaling in granular rheology across flow geometries," Phys. Rev. Lett. 125, 088002 (2020).
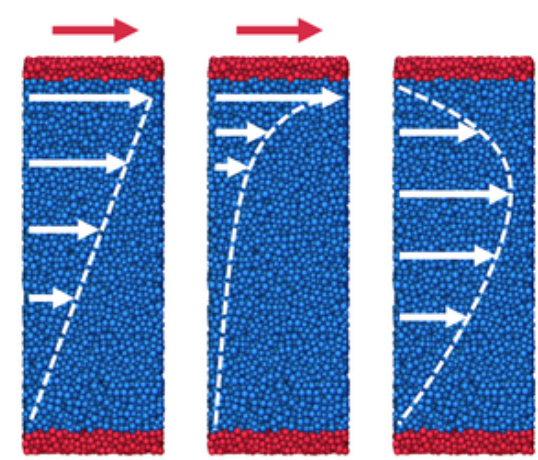

Credit: S. Kim and K. Kamrin [1] 